\title{
Complications with moderate-to-severe COVID-19 during hospital admissions in patients with pneumonia
}

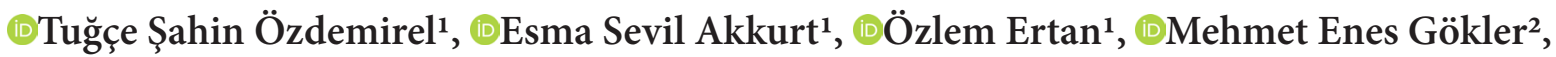 \\ ๑Berna Akıncı Özyürek ${ }^{1}$ \\ ${ }^{1}$ Health Sciences University, Ankara Atatürk Chest Diseases and Chest Surgery Training and Research Hospital, Department of Chest Disease, \\ Ankara, Turkey \\ ${ }^{2}$ Yıldırım Beyazıt University, Faculty of Medicine, Department of Public Health, Ankara, Turkey
}

Cite this article as: Şahin Özdemirel T, Akkurt ES, Ertan Ö, Gökler ME, Akıncı Özyürek B. Complications with moderate-to-severe COVID-19 during hospital admissions in patients with pneumonia. J Health Sci Med 2021; 4(6): 766-771.

\begin{abstract}
Introduction: Coronavirus disease 2019 (COVID-19), caused by SARS-CoV-2 (severe acute respiratory syndrome coronavirus 2) is an infectious disease that has caused significant mortality and morbidity worldwide. COVID-19 is known to cause complications, such as myocardial damage, acute coronary syndrome, deep vein thrombosis, pulmonary embolism, arrhythmia, heart failure, acute ischemic stroke, liver damage, cytokine storms, ischemia-reperfusion damage and side effects of drug treatments. In our study, we aimed to evaluate the complications that developed during hospitalizations in patients with moderate-to-severe COVID-19 related pneumonia who were hospitalized in our COVID-19 service.

Material and Method: This study included patients with moderate-to-severe COVID-19 pneumonia with a positive reverse transcriptase polymerase chain reaction (RT-PCR) test who were treated in our COVID-19 service between November 2020 and January 2021. Their demographic characteristics, treatment regimens, baseline laboratory values and complications during their hospitalization were retrospectively recorded.

Results: The study group had a mean age of $62.92 \pm 14.60$ years and $40.6 \%(n=55)$ were female. Approximately $35.3 \%(n=48)$ of the patients developed complications due to COVID-19 during their follow-up period. Of the patients with complications, $63.8 \%$ (n: 30 ) were male, most common complications were elevated liver enzymes (47.9\%) and pulmonary thromboembolism (20.8\%). Of those patients with complications, the most common comorbidities were hypertension ( $40.4 \%$ [ $n=19]$ ), diabetes mellitus $(25.5 \%[n=12])$ and cardiovascular disease $(23.4 \%[n=11])$. There were no significant relationships between the presence of complications and age, sex or comorbid diseases ( $p>0.05$ for each).

Conclusion: There are more underlying conditions, such as hypertension, diabetes mellitus, cardiovascular disease, chronic lung disease in hospitalized patients with moderate-to-severe pneumonia compared to outpatients with COVID-19 pneumonia. Complications develop, especially in the management of COVID-19 pneumonia, which affect the treatment process and patient mobilization.
\end{abstract}

Keywords: COVID-19, pneumonia, complication

\section{INTRODUCTION}

Coronavirus disease 2019 (COVID-19), caused by SARSCoV-2 (severe acute respiratory syndrome coronavirus 2), began in China at the end of 2019. COVID-19 is an infectious disease that has caused significant mortality and morbidity worldwide. Although it progresses with mild symptoms in most patients, serious complications, such as a cytokine storm, multiple organ failure, septic shock and acute respiratory distress syndrome (ARDS) have been observed in some cases (1-3).
COVID-19 is known to cause complications, such as myocardial damage, myocarditis, acute coronary syndrome, deep vein thrombosis, pulmonary embolism, arrhythmia, heart failure, acute ischemic stroke and cardiogenic shock $(4,5)$. In addition, it has been shown to cause liver damage by systemic inflammatory responses, cytokine storms, ischemia-reperfusion damage and side effects of drug treatments. The liver damage may also stem from an underlying liver disease or by direct actions 
on liver cells via angiotensin converting enzyme (ACE) receptors (6). Furthermore, the risk of a spontaneous pneumothorax is increased in patients with COVID-19, with an average incidence of approximately $1 \%(7,8)$.

In our study, we aimed to evaluate the complications that developed during hospitalizations in patients with moderate-to-severe COVID-19related pneumonia who were hospitalized and treated in our COVID-19 service.

\section{MATERIAL AND METHOD}

The study was conducted in compliance with the criteria of the Helsinki Declaration. It was approved by the Ethics Committee of the University of Health Sciences, Kecioren Training and Research Hospital (Date: 2021, Decision No: 2366). Written informed consent was obtained from all participants who participated in this study. Included in this study were patients with moderate-tosevere COVID-19 pneumonia with a positive reverse transcriptase polymerase chain reaction (RT-PCR) test who were treated in our COVID-19 service between November 2020 and January 2021. We followed the Republic of Turkey Ministry of Health COVID-19 Diagnosis and Treatment Guidelines to classify our patients as having moderate-to-severe pneumonia. This included patients with tachypnoea (respiratory rate $>30 /$ $\mathrm{min}$ ), poor prognostic criteria in blood tests and oxygen saturation levels $<90 \%$ in room air or at admission. Other criteria included a blood lymphocyte count $<800 /$ $\mu \mathrm{l}$; C-reactive protein $(\mathrm{CRP})>40 \mathrm{mg} / \mathrm{L}$; ferritin $>500 \mathrm{ng} /$ $\mathrm{ml}$; or D-dimer $>1.000 \mathrm{ng} / \mathrm{ml}$. Additionally, patients with bilateral pneumonia, as determined with a chest X-ray or a thoracic computed tomography, were recorded as cases with moderate-to-severe pneumonia. Demographic characteristics, treatment regimens, baseline laboratory values and complications during their hospitalization of all patients were recorded retrospectively. Among the complications were elevations in the liver enzymes alanine aminotransferase (ALT) and aspartate aminotransferase (AST) that exceeded the upper limits of the normal values.

\section{Statistical Analysis}

Data were evaluated using the IBM-SPSS (version 20.0) statistical package. For descriptive statistics, the following values were used: number, percentage, mean, standard deviation (SD), median and interquartile range (IQR: 25-75). Chi-square tests were used to compare categorical data, while Mann-Whitney U tests were used to compare continuous data. A logistic regression model was created with variables found to be significant in the bivariate analysis $(\mathrm{p}<0.05)$. Binary logistic regression analyses (were used in the model analysis. For statistical significance, $\mathrm{p}<0.05$ was accepted.

\section{RESULTS}

The study group had a mean age of $62.92 \pm 14.60$ years and $40.6 \%(\mathrm{n}=55)$ were female.. Approximately $35.3 \%$ $(n=48)$ of the patients developed complications due to COVID-19 during their follow-up period. Of the patients with complications, the two most common complications were elevated liver enzymes (47.9\%) and pulmonary thromboembolism $(20.8 \%)$ in $63.8 \% \quad(n=30)$ of the patients (Table 1). Of those patients with complications, the most common comorbidities were hypertension $(40.4 \%[\mathrm{n}=19])$, diabetes mellitus $(25.5 \%[\mathrm{n}=12])$ and cardiovascular disease $(23.4 \%[\mathrm{n}=11])$. While $14.9 \%(\mathrm{n}=7)$ of those with complications were diagnosed with chronic obstructive pulmonary disease (COPD), 10.6\% $(n=5)$ were diagnosed with asthma. There were no significant relationships between the presence of complications and age, sex or comorbid diseases ( $p>0.05$ for each). Table 2 shows the sociodemographic characteristics of the study group based on the presence of complications.

\begin{tabular}{|lcc|}
\hline \multicolumn{3}{|c|}{ Table 1. Distribution of complications seen in the study group } \\
\hline Complication & n & $\%$ \\
\hline Acute artery thombosis & 1 & 2.1 \\
Acute renal failure & 2 & 4.2 \\
Bradicardy & 1 & 2.1 \\
Deep vein thrombosis & 1 & 2.1 \\
Elevations of liver enzymes & 23 & 47.9 \\
Hydrocephalia & 1 & 2.1 \\
Pneumotorax & 4 & 8.4 \\
Pulmonary thromboembolism & 10 & 20.8 \\
Otitis media & 3 & 6.3 \\
Cerebrovascular event & 1 & 2.1 \\
New diabetes mellitus & 1 & 2.1 \\
Total & 48 & 100.0 \\
\hline
\end{tabular}

\begin{tabular}{|c|c|c|c|c|c|c|}
\hline & & \multicolumn{4}{|c|}{ Complication } & \multirow{3}{*}{$\mathbf{p}$} \\
\hline & & \multicolumn{2}{|c|}{ No } & \multicolumn{2}{|c|}{ Yes } & \\
\hline & & $\mathbf{n}$ & $\%$ & $\mathbf{n}$ & $\%$ & \\
\hline \multirow{2}{*}{ Gender } & Male & 49 & 57.0 & 30 & 63.8 & \multirow{2}{*}{0.442} \\
\hline & Female & 37 & 43.0 & 18 & 36.2 & \\
\hline \multirow{2}{*}{ Age } & $<65$ Age & 42 & 48.8 & 24 & 51.1 & \multirow{2}{*}{0.806} \\
\hline & $\geq 65$ Age & 44 & 51.2 & 23 & 48.9 & \\
\hline \multirow{2}{*}{ COPD } & No & 72 & 83.7 & 40 & 85.1 & \multirow{2}{*}{0.834} \\
\hline & Yes & 14 & 16.3 & 7 & 14.9 & \\
\hline \multirow{2}{*}{ Asthma } & No & 78 & 90.7 & 42 & 89.4 & \multirow{2}{*}{0.804} \\
\hline & Yes & 8 & 9.3 & 5 & 10.6 & \\
\hline \multirow{2}{*}{ Hypertension } & No & 42 & 48.8 & 28 & 59.6 & \multirow{2}{*}{0.236} \\
\hline & Yes & 44 & 51.2 & 19 & 40.4 & \\
\hline \multirow{2}{*}{$\begin{array}{l}\text { Diabetes } \\
\text { mellitus }\end{array}$} & No & 53 & 61.6 & 35 & 74.5 & \multirow{2}{*}{0.135} \\
\hline & Yes & 33 & 38.4 & 12 & 25.5 & \\
\hline \multirow{2}{*}{ Malignancy } & No & 74 & 86.0 & 43 & 91.5 & \multirow{2}{*}{0.356} \\
\hline & Yes & 12 & 14.0 & 4 & 8.5 & \\
\hline \multirow{2}{*}{ Hypotyroid } & No & 80 & 93.0 & 45 & 95.7 & \multirow{2}{*}{0.528} \\
\hline & Yes & 6 & 7.0 & 2 & 4.3 & \\
\hline \multirow{2}{*}{$\begin{array}{l}\text { Romatological } \\
\text { disease }\end{array}$} & No & 82 & 95.3 & 44 & 93.6 & \multirow{2}{*}{0.669} \\
\hline & Yes & 4 & 4.7 & 3 & 6.4 & \\
\hline \multirow{2}{*}{$\begin{array}{l}\text { Cardiovascular } \\
\text { disease }\end{array}$} & No & 63 & 73.3 & 36 & 76.6 & \multirow{2}{*}{0.673} \\
\hline & Yes & 23 & 26.7 & 11 & 23.4 & \\
\hline
\end{tabular}


Although $38.3 \%(n=18)$ of the patients who experienced complications had previously been admitted to intensive care, $34 \%(n=16)$ were on pulse steroids. Pulse steroid use and the presence of desaturation were more frequent in those with complications ( $\mathrm{p}=0.012, \mathrm{p}=0.009$, respectively).

In our study, we observed that 129 of 134 patients were given low molecular weight heparin (LMWH) treatment as venous thromboembolism prophylaxis. No relationship was found between the use of LMWH, acetylsalicylic acid (ASA), colchicine, ground glass in radiological imaging, the presence and extent of consolidation and the development of complications ( $p>0.05$ for each). Table 3 shows the need for intensive care, drug use and radiological characteristics of the study group based on the presence of complications.

In the group with complications, the length of stay was significantly longer than in the group without complications $(\mathrm{p}=0.001)$. White blood cells $(\mathrm{WBC})$, lymphocytes, platelets (PLT), albumin, lactate dehydrogenase (LDH), CRP, troponin, ferritin, $\mathrm{D}$-dimer, pro-brain natriuretic peptide (pro-BNP) and haemoglobin $\mathrm{Alc}(\mathrm{HgAlc})$ values were evaluated according to the presence of complications in the study community. There was no correlation between the presence of complications and these parameters $(p>0.05$ in each case). Table 4 shows the age, duration of hospital stay and laboratory values of the sample group based on the occurrence of complications.
The length of hospital stay (OR: 1.066, 95\% CI: 1.0041.132) was a risk factor for the existence of complications when the significant variables were analysed according to the presence of complications in the logistic regression analysis (Table 5).

\begin{tabular}{|c|c|c|c|c|c|c|}
\hline \multicolumn{7}{|c|}{$\begin{array}{l}\text { Table } 3 \text {. Intensive care need, drug use and radiological characteristics } \\
\text { of the study group according to the presence of complications }\end{array}$} \\
\hline & & \multicolumn{4}{|c|}{ Complication } & \multirow{3}{*}{ p } \\
\hline & & \multicolumn{2}{|c|}{ No } & \multicolumn{2}{|c|}{ Yes } & \\
\hline & & $\mathbf{n}$ & $\mathbf{n}$ & $\mathbf{n}$ & $\mathbf{n}$ & \\
\hline \multirow{2}{*}{ ICU } & No & 56 & 65.1 & 29 & 61.7 & \multirow{2}{*}{0.695} \\
\hline & Yes & 30 & 34.9 & 18 & 38.3 & \\
\hline \multirow{2}{*}{$\begin{array}{l}\text { Use of pulse } \\
\text { steroid }\end{array}$} & No & 73 & 84.9 & 31 & 66.0 & \multirow{2}{*}{0.012} \\
\hline & Yes & 13 & 15.1 & 16 & 34.0 & \\
\hline \multirow{2}{*}{ Desaturation } & No & 24 & 27.9 & 4 & 8.5 & \multirow{2}{*}{0.009} \\
\hline & Yes & 62 & 72.1 & 43 & 91.5 & \\
\hline \multirow{2}{*}{ LMWH } & No & 3 & 3.5 & 1 & 2.1 & \multirow{2}{*}{0.661} \\
\hline & Yes & 83 & 96.5 & 46 & 97.9 & \\
\hline \multirow{2}{*}{$\begin{array}{l}\text { Acetylsalicilic } \\
\text { acid }\end{array}$} & No & 59 & 68.6 & 31 & 66.0 & \multirow{2}{*}{0.755} \\
\hline & Yes & 27 & 31.4 & 16 & 34.0 & \\
\hline \multirow{2}{*}{ Colchicine } & No & 72 & 83.7 & 33 & 70.2 & \multirow{2}{*}{0.068} \\
\hline & Yes & 14 & 16.3 & 14 & 29.8 & \\
\hline \multirow{2}{*}{$\begin{array}{l}\text { Ground glass } \\
\text { opacity }\end{array}$} & No & 7 & 8.1 & 3 & 6.4 & \multirow{2}{*}{0.713} \\
\hline & Yes & 79 & 91.9 & 44 & 93.6 & \\
\hline \multirow{2}{*}{ Consolidation } & No & 32 & 37.2 & 11 & 23.4 & \multirow{2}{*}{0.104} \\
\hline & Yes & 54 & 62.8 & 36 & 76.6 & \\
\hline \multirow{2}{*}{$\begin{array}{l}\text { Radiological } \\
\text { infiltration }\end{array}$} & $<\% 50$ & 54 & 62.8 & 22 & 46.8 & \multirow{2}{*}{0.075} \\
\hline & $>50$ & 32 & 37.2 & 25 & 53.2 & \\
\hline \multirow{2}{*}{ Final } & Alive & 77 & 89.5 & 42 & 89.4 & \multirow{2}{*}{0.975} \\
\hline & Exitus & 9 & 10.5 & 5 & 10.6 & \\
\hline
\end{tabular}

\begin{tabular}{|c|c|c|c|c|c|c|c|}
\hline & \multicolumn{6}{|c|}{ Complication } & \multirow{3}{*}{$\mathbf{p}$} \\
\hline & \multicolumn{3}{|c|}{ No } & \multicolumn{3}{|c|}{ Yes } & \\
\hline & median & IQR 25 & IQR 75 & median & IQR 25 & IQR 75 & \\
\hline Age & 65.0 & 55.0 & 75.0 & 64.0 & 53.0 & 73.0 & 0.675 \\
\hline Length of hospital stay & 9.0 & 6.0 & 14.0 & 14.0 & 8.0 & 19.0 & 0.001 \\
\hline Length of ICU stay & 6.0 & 2.0 & 9.0 & 9.0 & 5.0 & 14.0 & 0.083 \\
\hline WBC & 7595.0 & 5680.0 & 10620.0 & 8465.0 & 6120.0 & 11980.0 & 0.234 \\
\hline Lymphocyte & 1015.0 & 690.0 & 1780.0 & 955.0 & 700.0 & 1420.0 & 0.599 \\
\hline Lymphocyte \% & 16.1 & 8.4 & 24.6 & 12.2 & 7.8 & 18.7 & 0.099 \\
\hline Platelet & 239.0 & 186.0 & 301.0 & 245.5 & 190.0 & 330.0 & 0.474 \\
\hline Albumin & 33.6 & 29.0 & 37.5 & 31.6 & 29.8 & 34.7 & 0.333 \\
\hline $\mathrm{LDH}$ & 295.5 & 217.0 & 423.5 & 342.0 & 280.0 & 487.0 & 0.106 \\
\hline CRP & 86.5 & 28.0 & 145.0 & 99.9 & 47.0 & 186.8 & 0.166 \\
\hline Troponin & 5.9 & 3.2 & 17.3 & 7.7 & 4.1 & 20.6 & 0.413 \\
\hline Ferritin & 226.5 & 91.8 & 555.0 & 368.1 & 220.2 & 589.4 & 0.085 \\
\hline D-dimer & .8 & .4 & 1.5 & 1.0 & .6 & 2.7 & 0.080 \\
\hline Pro-BNP & 65.9 & 35.9 & 133.0 & 58.6 & 30.9 & 198.8 & 0.904 \\
\hline $\mathrm{HgAlC}$ & 7.0 & 5.8 & 9.7 & 6.9 & 6.4 & 7.9 & 0.850 \\
\hline Number of lung lobes affected & 4.0 & 3.0 & 5.0 & 5.0 & 3.0 & 5.0 & 0.079 \\
\hline
\end{tabular}

\begin{tabular}{|c|c|c|c|c|c|}
\hline & $\beta$ & S.E. & p & OR & $\% 95 \mathrm{CI}$ \\
\hline The length of hospital stay & 0.064 & 0.031 & 0.036 & 1.066 & $1.004-1.132$ \\
\hline Use of pulse steroid & 0.298 & 0.509 & 0.558 & 1.347 & $0.497-3.656$ \\
\hline Desaturation & 0.985 & 0.642 & 0.125 & 2.678 & $0.761-9.421$ \\
\hline
\end{tabular}




\section{DISCUSSION}

In our study, 134 patients who were RT-PCR positive, had moderate-to-severe COVID-19 pneumonia and were hospitalized between November 2020 and January 2021 were analysed retrospectively. The patients were divided into two groups: those with complications and those without complications. Males comprised 59.3\% of our hospitalized COVID-19 patients and 63.8\% $(n=30)$ of the patients who developed complications. Previous studies have shown that men experience a more severe disease and have a higher mortality rate compared to women (9). Higher rates of comorbidities, such as hypertension (HT) and diabetes mellitus (DM), are also observed in patients hospitalized for severe disease (1). The most common comorbidities in our study were HT and DM. However, no significant effects of comorbidities on the development of complications were found. Lymphopenia is seen with COVID-19 as in other viral infections. The severity of lymphopenia is related to the severity of the disease and is effective in the decision of hospitalization $(7,10)$. In our study, lymphopenia was present in most of the patients, but no significant relationship was found between this condition and the development of complications.

Moderate liver enzyme elevations (especially AST and ALT) are common in patients with COVID-19. These may be associated with severe disease and increased inflammation, but they generally do not result in liver dysfunction; thus, no liver-directed therapy is required (11). Liver enzyme levels vary between $4 \%-33 \%$ in patients with COVID-19; however, this rate has reached $39 \%$ in some studies (12-14). In our study, liver enzyme elevations were observed in 47 patients (47.9\% of patients with complications, $35.3 \%$ of all patients). The most frequently reported adverse events related to Favipiravir use in clinical studies are increased serum uric acid levels, diarrhoea, decreased neutrophil counts and liver enzyme elevations. In light of these findings, it was difficult to determine if the increases in liver enzymes in our patients were due to the medication or to the moderate-to-severe COVID-19 pneumonia. In patients with COVID-19, pulmonary thromboembolisms occur at a rate of $10 \%-28 \%$, with the average age of onset being 57-61 years $(15,16)$. In a study by Bompard et al. (17), the cumulative rate for thromboembolism was $24 \%$ and $50 \%$ in COVID-19 patients followed in intensive care.

D-dimer levels are significantly higher in a group with thromboembolisms (17). In another study, D-dimer values were associated with acute thrombosis, and generally $>1$ IU was found to be significant $(18,19)$. In two separate studies evaluating 314 and 124 patients with COVID-19 who were hospitalized outside the intensive care unit, the venous thromboembolism
(VTE) rate was $6.4 \%$ and $3 \%$, respectively $(20,21)$. In our study, the pulmonary thromboembolism (PTE) rate was $7.5 \%$ for all patients and $20.8 \%$ in the group with complications. D-dimer values were also higher in the group with complications, but not significantly higher, and a threshold $\mathrm{D}$-dimer value could not be defined for determining the risk of PTE. This is due to the fact that all of our patients were hospitalized with a diagnosis of moderate-to-severe pneumonia. This suggests that high D-dimer levels (> $0.5 \mathrm{IU}$ ) may indicate an increased risk of PTE, as well as be associated with the severity of pneumonia. We think our PTE rate was lower than that in the literature since all our patients used prophylactic LMWH.

Studies have shown that COVID-19 has a 1\% rate of causing a spontaneous pneumothorax. There are also reports that it is seen less frequently $(7,8)$. The mechanism of a pneumothorax in COVID-19 infections remains unclear. Risk factors for a spontaneous pneumothorax include male sex, slim and tall body build, smoking, trauma and infection. In our study, the four patients who developed a pneumothorax were male. Parenchymal lesions, such as cysts and bullae, were not found in the first thoracic CT images. In the literature, a case with a pneumothorax due to a giant bulla (22) and a case with cystic lung lesions causing a pneumothorax, both resulting from of a COVID-19 infection, were published by Liu K et al. (23). In a study with 3,000 patients, Massa Zantah et al. (8) reported the presence of lymphopenia and increased inflammatory markers, such as CRP, LDH, ferritin, D-dimer and interleukin 6 (IL-6) in almost all patients who developed a spontaneous pneumothorax. When a pneumothorax developed in our patients, inflammatory parameters were high and consistent with the literature, but no significant relationships were found with the development of complications.

In COVID-19, as in other viral infections, there is a risk of infectious particles spreading from the nasopharynx to the middle ear. Viral infections may lead to otitis media either as a single middle ear pathogen or by causing a eustachian tube dysfunction. There have been several cases with otitis media detected during the course of COVID-19 $(24,25)$. In our study, otitis media was detected in three patients $(2.2 \%$ of all patients and $6.3 \%$ of those with complications).

Mao et al. (26) reported that cerebrovascular disease develops in $5.7 \%$ of patients with severe infections. In another study by Li Y et al. (27), the incidence of cerebrovascular events in patients with COVID-19 was approximately $5 \%$ with a mean age of 71.6 years. In our study, this rate was $2.1 \%$ in patients with complications, and $0.7 \%$ in the entire study group. 
The duration of hospital stay was higher in the group with complications than in the group without complications. This suggests that, with more immobilization, the higher the risk of complications.

The limitations of our study include the limited number of patients in our study, the evaluation of only inpatients, inadequate follow up after discharge and the retrospective nature of the study.

\section{CONCLUSION}

The main points in our study are: The most common complications in our inpatients with covid 19 pneumonia; liver enzyme elevation and pulmonary thromboembolism were consistent with the literature. It was observed that the most common underlying diseases in the development of complications in COVID-19 patients were hypertension, diabetes mellitus and cardiovascular diseases, again in line with the literature. The duration of hospital stay was higher in the group with complications compared to the group without complications. It showed that we should be careful in terms of complication development as the immobilization time gets longer.

There are more underlying conditions, such as hypertension, diabetes mellitus, cardiovascular disease, chronic kidney disease or chronic lung disease in hospitalized patients with moderate-to-severe pneumonia compared to outpatients with COVID-19 pneumonia. Hypertension is the most common underlying condition in patients with moderate-to-severe COVID-19 pneumonia. Complications develop, especially in the management of COVID-19 pneumonia, which affect the treatment process and patient mobilization. Therefore, it is important to perform laboratory follow up in the clinical follow up and to be especially careful in terms of the development of coagulopathy, pneumothorax and neurological events.

\section{ETHICAL DECLARATIONS}

Ethics Committee Approval: It was approved by the Ethics Committee of the University of Health Sciences, Kecioren Training and Research Hospital (Date: 2021, Decision No: 2366).

Informed Consent: Written informed consent was obtained from all participants who participated in this study.

Referee Evaluation Process: Externally peer-reviewed.

Conflict of Interest Statement: The authors have no conflicts of interest to declare.

Financial Disclosure: The authors declared that this study has received no financial support.
Author Contributions: All of the authors declare that they have all participated in the design, execution, and analysis of the paper, and that they have approved the final version.

\section{REFERENCES}

1. Zhou F, Yu T, Du R, et al. Clinical course and risk factors for mortality of adult inpatients with CO- VID-19 in Wuhan, China: a retrospective cohort study. Lancet 2020; 395: 1054-62.

2. Du Toit A. Outbreak of a novel coronavirus. Nat Rev Microbiol 2020; 18: 123 .

3. European Centre for Disease Prevention and Control. Q \& A on COVID-19: Basic facts. Available from: https://www. ecdc.europa. eu/en/covid-19/facts/questions-answersbasic-facts Accessed date: 18 Sept 2020.

4. Mahajan P, Dass B, Radhakrishnan N, McCullough PA. COVID19-Associated Systemic Thromboembolism: A Case Report and Review of the Literature. Cardiorenal Med 2020; 10: 462-9.

5. Boukhris M, Hillani A, Moroni F, et al. Cardiovascular Implications of the COVID-19 Pandemic: A Global Perspective. Can J Cardiol 2020; 36: 1068-80.

6. Tian D, Ye Q. Hepatic complications of COVID-19 and its treatment. J Med Virol 2020; 92: 1818-24.

7. Chen N, Zhou M, Dong X, et al. Epidemiological and clinical characteristics of 99 cases of 2019 novel coronavirus pneumonia in Wuhan, China: a descriptive study. Lancet 2020; 395: 507-13.

8. Zantah M, Dominguez Castillo E, Townsend R, Dikengil F, Criner GJ. Pneumothorax in COVID-19 disease- incidence and clinical characteristics. Respir Res 2020; 21: 236.

9. Jin JM, Bai P, He W, et al. Gender differences in patients with COVID-19: focus on severity and mortality. Front Public Health 2020; 8: 152.

10. Qiu P, Zhou Y, Wang F, et al. Clinical characteristics, laboratory outcome characteristics, comorbidities, and complications of related COVID-19 deceased: a systematic review and metaanalysis. Aging Clin Exp Res 2020; 32: 1869-78.

11. Bertolini A, Van de Peppel IP, Bodewes FAJA, Moshage H, Fantin A, Farinati F. Abnormal liver function tests in patients with COVID-19: Relevance and Potential Pathogenesis. Hepatology 2020; 72: 1864-72.

12.Zhang Y, Zheng L, Liu L, Zhao M, Xiao J, Zhao Q. Liver impairment in COVID-19 patients: A retrospective analysis of 115 cases from a single centre in Wuhan city, China. Liver Int 2020; 40: 2095-103.

13. Cai Q, Huang D, Yu H, et al. COVID-19: Abnormal liver function tests. J Hepatol 2020; 73: 566-74.

14. Fan Z, Chen L, Li J, et al. Clinical features of COVID-19-related liver damage. Clin Gastroenterol Hepatol 2020; 18: 1561-6.

15. Porfidia A, Valeriani E, Pola R, Porreca E, Rutjes A, Di Nisio M. Venous thromboembolism in patients with COVID-19: Systematic review and meta-analysis. Thrombosis research 2020; 196: 67-74.

16. Boonyawat K, Chantrathammachart P, Numthavaj P, et al. Incidence of thromboembolism in patients with COVID-19: a systematic review and meta-analysis. Thrombosis Journal 2020: $23 ; 18: 34$.

17. Bompard F, Monnier H, Saab I et al. Pulmonary embolism in patients with COVID-19 pneumonia. Eur Respir J. 2020; 56: 2001365.

18.Xu Y, Qian Y, Gu Q, et al. Relationship between d-dimer concentration and inflammatory factors or organ function in patients with coronavirus disease 2019. Zhonghua Wei Zhong Bing Ji Jiu Yi Xue 2020; 32: 559-63.

19. Li Y, Zhao K, Wei H, et al. Dynamic relationship between d-dimer and COVID-19 severity. Br J Haematol 2020; 190: e24-7. 
20. Middeldorp S, Coppens M, van Haaps TF, et al. Incidence of venous thromboembolism in hospitalized patients with COVID-19. J Thromb Haemost. 2020 Aug;18(8):1995-2002.

21.Lodigiani C, Iapichino G, Carenzo L, et al. Venous and arterial thromboembolic complications in COVID-19 patients admitted to an academic hospital in Milan, Italy. Thromb Res 2020; 191: 9.

22. Sun R, Liu H, Wang X. Mediastinal emphysema, Giant Bulla and pneumothorax developed during the course of COVID-19 pneumonia. Korean J Radiol 2020; 21: 541-44.

23. Liu K, Zeng Y, Xie P, et al. COVID-19 with cystic features on computed tomography: A case report. Medicine (Baltimore) 2020; 99: e20175.

24. Mohan S, Workman A, Barshak M, Welling DB, Abdul-Aziz D. Considerations in Management of Acute Otitis Media in the COVID-19 Era. Ann Otol Rhinol Laryngol 2021; 130: 520-27.

25. Fidan V. New type of corona virus induced acute otitis media in adult. Am J Otolaryngol 2020; 41: 102487.

26. Mao L, Jin $H$, Wang $M$, et al. Neurologic manifestations of hospitalized patients with coronavirus disease 2019 in Wuhan, China. JAMA Neurol 2020; 77: 683-90.

27.Li Y, Li M, Wang M, et al. Acute cerebrovascular disease following COVID-19: a single center, retrospective, observational study. Stroke Vasc Neurol 2020; 5: 279-84. 